$\underline{\text { Original Articles }}$

\title{
Prevalence of comorbidities in children with attention deficit and hyperactivity disorder at Lady Ridgeway Hospital for Children, Sri Lanka
}

\author{
*G S Wijetunge ${ }^{1}$, J C Dayasena ${ }^{2}$, I C Kulathilake ${ }^{2}$, P Ratnathilake ${ }^{3}$, Y Namasivayam ${ }^{4}$ \\ Sri Lanka Journal of Child Health, 2015: 44(2): 77-81
}

\begin{abstract}
Background: Attention deficit and hyperactivity disorder (ADHD) has a high prevalence and is frequently associated with comorbid illnesses.

Objective: To assess the comorbid patterns, sociodemographic profiles, management patterns and outcomes amongst children with ADHD presenting to the Child and Adolescent Mental Health Services at the Lady Ridgeway Hospital for Children.
\end{abstract}

Method: Two hundred patients, aged 6-12 years, diagnosed with ADHD, were assessed for their demographic features and comorbidities based on DSM IV criteria.

Results: Of the 200 children, 166 (83\%) were male and 198 (99\%) were schooling. While 108 (54\%) had a single neuropsychiatric comorbidity, $30(15 \%)$ had 2 comorbidities and $1(0.5 \%)$ had 3 comorbidities. Specific developmental disorder of scholastic skills (SDDSS) was the most prevalent comorbidity and was seen in $90(45 \%)$ patients. Oppositional defiant disorder (ODD) was seen in $56(28 \%)$ children and $17(8.5 \%)$ had both SDDSS and ODD. In the sample, $90 \%$ of children were born of uneventful deliveries. Postnatal complications were found in $12 \%$ children. In the sample, $30 \%$ had a history of febrile fits and $2.5 \%$ had a history of afebrile fits.

Conclusions: In this sample of 200 children with diagnosed ADHD, 139 (69.5\%) had one or more neuropsychiatric comorbidities. SDDSS was the most prevalent comorbidity

${ }^{1}$ Child and Adolescent Psychiatrist, Lady Ridgeway Hospital for Children (LRHC), Colombo, ${ }^{2}$ Medical Officer Psychiatry. National Hospital, Sri Lanka, ${ }^{3}$ Medical Officer Child and Adolescent Mental Health Services, LRHC, Colombo, ${ }^{4}$ Medical Officer Laboratory, National Hospital, Sri Lanka

*Correspondence: swarnawije@gmail.com

(Received on 10 June 2014: Accepted after revision on 2 August 2014)
(Key words: Attention deficit and hyperactivity disorder, specific developmental disorder of scholastic skills, conduct disorder, oppositional defiant disorder, comorbidity)

\section{Background}

Attention deficit hyperactivity disorder (ADHD) is defined in DSM IV as a condition having core symptoms of hyperactivity, impulsivity and inattentiveness, which are developmentally inappropriate and give rise to impairment in function. The symptoms have to be present in more than one setting, usually home and school ${ }^{1}$. It is difficult to establish a diagnosis of ADHD in children younger than 4 years as their normal behaviour is more variable than that of older children ${ }^{2}$. Twin studies show that ADHD has a polygenic inheritance in about $80 \%$ of patients, making it one of the most strongly familial neurodevelopmental disorders ${ }^{3}$. Based on ICD 10 criteria, the prevalence of ADHD is around 1 to $2 \%$ for school children, and according to DSM IV criteria it reaches $5 \%$, the prevalence rate varying from 2.4 to $9 \%$ depending on how strictly the impairment is defined ${ }^{2}$. In more than half the patients diagnosed with ADHD in childhood, core symptoms can persist into adolescence ${ }^{4}$.

A descriptive study of attention deficit hyperactivity disorder (ADHD) at the Lady Ridgeway Hospital for Children, Colombo, was conducted in 2002. Diagnosis was made according to DSM-IV criteria and 37 out of 517 children attending child guidance clinic at LRH had ADHD. There was a male preponderance. Mean age was 6.5 years. Learning disorders and obsessive compulsive disorders / behaviours were the commonly associated neuropsychiatric conditions ${ }^{5}$.

In the US National Comorbidity Survey replication of 2006, 50-80\% children with ADHD had an associated illness ${ }^{6}$. ADHD is known to be comorbid with disorders such as specific developmental disabilities of scholastic skills (SDDSS), conduct disorder (CD), oppositional defiant disorder (ODD), movement 
Prevalence of comorbidities in children with attention deficit and hyperactivity disorder at Lady Ridgeway Hospital for Children, Sri Lanka Sri Lanka J. Child Health 2015; 44(2): 77-81

disorders (e.g. tics), adjustment disorders, obsessive compulsive disorder (OCD), anxiety disorders and mood disorders ${ }^{6}$.

\section{Objectives}

To assess the comorbid patterns, socio-demographic profiles, management patterns and outcomes amongst children with ADHD presenting to the Child and Adolescent Mental Health Services (CAMHS) at the Lady Ridgeway Hospital (LRH) for Children, Colombo.

\section{Method}

A hospital based, cross sectional descriptive study was carried out at LRH, the largest tertiary care paediatric hospital in Sri Lanka. The CAMHS clinic at LRH is the largest child psychiatry clinic in Sri Lanka. Children from all districts of Sri Lanka are referred to this clinic. Children from government hospitals including outpatient departments and wards of LRH, government clinics, private sector hospitals, private sector clinics, schools, probation facilities and children's homes are referred to the CAMHS clinic. Traditionally the patient population of LRH comprises children below the age of 12 years. Ethical committee approval was obtained from the LRH Ethical Review Committee.

A consecutive sample of eligible children, who were diagnosed to have ADHD at the CAMHS clinic during the study period from 01.06.2012 to 01.05.2013, was included in the study. A sample of 196 children was expected to be adequate to have an at least $5 \%$ precision of estimated variables with a confidence level of $95 \%, 5 \%$ alpha error and expected proportion of $15 \%$. We included 200 children to keep allowances for missing data. Children identified to have had global developmental delay, children being treated for epilepsy and children having a history of substance abuse were excluded from the study. Informed consent was taken from the parent or guardian.

Collection of data was performed by the principal investigator and two interviewers using an interviewer administered questionnaire. Once the socio-demographic data was recorded, the child was observed and clinically assessed along with the parents or guardians. Other documentary information from the teachers such as SNAP IV forms completed by the teacher and the teacher's report on the child were also taken into account in order to confirm the diagnosis. In addition, child was assessed for the presence of other common neuropsychiatric comorbidities, including CD, ODD, anxiety disorder, OCD, SDDSS, tic disorder and depression. These comorbidities were diagnosed according to DSM IV criteria by the consultant psychiatrist. SDDSS was assessed using standardized clinic based assessment according to the National Institute of Education curricula. These comorbidities were chosen as they are known to commonly occur with ADHD. Data was processed using PASW 18 software. Prevalence of comorbidities was summarized according to their various combinations of presentations as well as number of comorbidities per child.

\section{Results}

Of the 200 children with ADHD, 84 (42\%) were between 6-8 years, 79 (39.5\%) between 8-10 years and $34(17 \%)$ were in the age group of $10-12$. Mean age of the sample was $8.32(\mathrm{SD}=1.8)$ years. One hundred and sixty six $(83 \%)$ patients were males. One hundred and eight (54\%) patients were from the Colombo District. One hundred and ninety eight (99\%) children were schooling. Ninety (45\%) fathers had studied up to ordinary level $(\mathrm{O} / \mathrm{L})$ and a further $50(25 \%)$ up to grade 10 . One hundred and thirty $(65 \%)$ children had fathers with semi-skilled or unskilled occupations. Of the mothers, 160 (80\%) were housewives and $16(8 \%)$ were semiskilled. One hundred and forty two (71\%) mothers were educated up to the secondary level. One hundred and eighty six (93\%) parents were married, 7 (3.5\%) were divorced and $7(3.5 \%)$ were separated.

One hundred and sixty six (83\%) mothers denied having any psychosocial issues among family members such as aggression, substance misuse and deliberate self-harm at the time of the interview. Only $12(6 \%)$ children had parents with consanguineous marriages. One hundred and thirty $(65 \%)$ patients did not report any family history of mental illness. However, 26 (13\%) had another family member with ADHD. Other neuropsychiatric disorders recorded in the family were SDDSS in $3(1.5 \%), \mathrm{CD} / \mathrm{ODD}$ in 1 $(0.5 \%)$, depression in $7(3.5 \%)$, anxiety in $2(1 \%)$, bipolar disorder in $1(0.5 \%)$ and learning disability in $3(1.5 \%)$. Antenatal problems were not seen in 168 (84\%) mothers whilst $18(9 \%)$ reported pregnancy induced hypertension and 7 (3.5\%) had gestational diabetes. Other prenatal complications included twins, past dates, hypothyroidism, insomnia, deliberate self-harm (drug overdose by mother) and marital problems.

One hundred and eighty (90\%) deliveries were uneventful whilst $20(10 \%)$ reported perinatal complications including birth asphyxia in $1(0.5 \%)$, 
Prevalence of comorbidities in children with attention deficit and hyperactivity disorder at Lady Ridgeway Hospital for Children, Sri Lanka Sri Lanka J. Child Health 2015; 44(2): 77-81

meconium aspiration in $5(2.5 \%)$, low birth weight in $5(2.5 \%)$, fetal distress in $1(0.5 \%)$ and prematurity in $2(1 \%)$. Other identified complications included malpresentation, premature rupture of membranes and cord around the neck in a small number of patients. Postnatally, $176(88 \%)$ patients did not have any ailment, $6(3 \%)$ had infections, $14(7 \%)$ had jaundice, $1(0.5 \%)$ had hypoglycaemia and $1(0.5 \%)$ had neonatal fits. Other complications included respiratory distress, septicaemia and pneumothorax in a small number of patients. One hundred and twenty six $(63 \%)$ patients had no significant medical illness in the past whilst $60(30 \%)$ had febrile fits and 5 $(2.5 \%)$ had afebrile fits. One hundred and forty $(70 \%)$ ADHD patients had one or more associated comorbidities as shown in Tables 1 and 2 .

Table 3 shows the distribution of children according to the type of neuropsychiatric comorbidities.

Table 1: Presence of comorbidities among ADHD children

\begin{tabular}{|l|c|}
\hline \multicolumn{1}{|c|}{ Comorbidity } & No. of children with comorbidity (\%) \\
\hline Specific developmental disorder of scholastic skills (SDDSS) & $90(45)$ \\
\hline Oppositional Defiant Disorder (ODD) & $46(23)$ \\
\hline Conduct Disorder (CD) & $08(04)$ \\
\hline Anxiety Disorder & $03(1.5)$ \\
\hline Obsessive Compulsive Disorder (OCD) & $05(2.5)$ \\
\hline Tics & $05(2.5)$ \\
\hline Depression & $02(01)$ \\
\hline Emotional/ adjustment disorder & $10(05)$ \\
\hline
\end{tabular}

Table 2: Distribution of children according to number of neuropsychiatric comorbidities

\begin{tabular}{|c|c|}
\hline Number of comorbidities & Number of children $(\%)$ \\
\hline Attention deficit and hyperactivity disorder only & $60(30)$ \\
\hline Single Comorbidity & $108(54)$ \\
\hline Two Comorbidities & $30(15)$ \\
\hline Three Comorbidities & $01(0.5)$ \\
\hline No Data & $01(0.5)$ \\
\hline Total & $200(100)$ \\
\hline
\end{tabular}

Table 3: Distribution of children according to type of neuropsychiatric comorbidities

\begin{tabular}{|l|l|c|}
\hline \multicolumn{2}{|c|}{ Type of Comorbidity/ Comorbidities } & No. of children (\%) \\
\hline \multirow{4}{*}{ One Comorbidity } & SDDSS & $63(31.5)$ \\
\cline { 2 - 3 } & ODD & $28(14)$ \\
\cline { 2 - 3 } & CD & $05(2.5)$ \\
\cline { 2 - 3 } & Anxiety disorder & $03(1.5)$ \\
\cline { 2 - 3 } & OCD & $02(01)$ \\
\cline { 2 - 3 } Two or three Comorbidities & Tic disorder & $02(01)$ \\
\cline { 2 - 3 } & Depression & $02(01)$ \\
\cline { 2 - 3 } & Emotional disorders & $03(1.5)$ \\
\hline \multirow{5}{*}{} & OCD + Tics & $01(0.5)$ \\
\cline { 2 - 3 } & SDDSS + Tics & $01(0.5)$ \\
\cline { 2 - 3 } & ODD + OCD & $01(0.5)$ \\
\cline { 2 - 3 } & SDDSS+ ODD & $17(8.5)$ \\
\cline { 2 - 3 } & OCD + SDDSS & $01(0.5)$ \\
\cline { 2 - 3 } & CD + SDDSS & $02(01)$ \\
\cline { 2 - 3 } & SDDSS + Emotional disorders & $05(2.5)$ \\
\cline { 2 - 3 } & CD + Emotional disorders & $01(0.5)$ \\
\cline { 2 - 3 } & SDDSS + Tics + Emotional disorders & $01(0.5)$ \\
\hline
\end{tabular}


Prevalence of comorbidities in children with attention deficit and hyperactivity disorder at Lady Ridgeway Hospital for Children, Sri Lanka Sri Lanka J. Child Health 2015; 44(2): 77-81

Ninety six percent of children were on one or more drugs. The majority were on methylphenidate with or without other drugs. There were 8 children who were on fluoxetine, with or without other drugs. Activities of daily living (ADL) was satisfactory in 95.5\%. Table 4 presents the educational performances among ADHD children.

Table 4: Distribution of children according to level of skills in reading, writing, mathematics and comprehension

\begin{tabular}{|l|c|c|c|c|}
\hline & $\begin{array}{c}\text { Reading } \\
\text { No. (\%) }\end{array}$ & $\begin{array}{c}\text { Writing } \\
\text { No. (\%) }\end{array}$ & $\begin{array}{c}\text { Mathematics } \\
\text { No. (\%) }\end{array}$ & $\begin{array}{c}\text { Comprehension } \\
\text { No. (\%) }\end{array}$ \\
\hline Average or above average & $98(49.0 \%)$ & $88(44.0 \%)$ & $81(40.5 \%)$ & $95(47.5 \%)$ \\
\hline Below average & $101(50.5 \%)$ & $111(55.5 \%)$ & $118(59.0 \%)$ & $104(52.0 \%)$ \\
\hline No data & $01(0.5 \%)$ & $01(0.5 \%)$ & $01(0.5 \%)$ & $01(0.5 \%)$ \\
\hline Total & $200(100 \%)$ & $200(100 \%)$ & $200(100 \%)$ & $200(100 \%)$ \\
\hline
\end{tabular}

\section{Discussion}

The presence of comorbid disorders requires special consideration in the treatment of patients with ADHD. There seems to be a direct relationship between the severity of the illness and the comorbid disorder. Conversely it has been observed that patients with ADHD and a comorbid disorder appear to have more severe ADHD symptoms with problems in diagnosis and treatment and hence both disorders have to be diagnosed and treated simultaneously ${ }^{6}$.

In our study $139 \quad(69.5 \%)$ patients had a neuropsychiatric comorbidity which is comparable to the result of the National Comorbidity Survey 2006 in the US, where $50-80 \%$ patients had an associated illness ${ }^{7}$. Whilst $108(54 \%)$ had a single comorbidity, $30(15 \%)$ had two and one $(0.5 \%)$ had three comorbidities. In our study $83 \%$ were males which is in accordance with a survey carried out in the Cambridge University in 2002 which showed that the disorder is 2-3 times more frequent in males than females in their community ${ }^{8}$. In the previous study carried out at LRH too, male predominance was a feature ${ }^{5}$.

SDDSS was the most prevalent comorbid disorder being found in $90(45 \%)$ patients. ODD was the commonest of the disruptive behavioural comorbidities unlike in the United Kingdom where $\mathrm{CD}$ is the commonest disruptive disorder ${ }^{9}$. According to past research, mothers of ADHD children are more likely to have experienced pregnancy complications and birth complications ${ }^{10}$. In our study there were no antenatal problems in $168(84 \%)$ mothers, whilst 18 (9\%) had pregnancy induced hypertension and 7 (14\%) had gestational diabetes. Whilst 180 (90\%) deliveries were uneventful, $20(10 \%)$ had suffered from birth asphyxia $(0.5 \%)$, meconium aspiration $(2.5 \%)$, low birth weight $(2.5 \%)$, fetal distress $(0.5 \%)$ and prematurity $(1 \%)$.
ADHD patients with concurrent active seizures on treatment were excluded from the study as the association of these two are quite complex. Poor scholastic skills, adjustment problems and disruptive behaviours can influence the long term achievements of these children.

In our study, patients were mostly given methylphenidate for symptom control. There is some evidence that the presence of anxiety as a comorbidity will reduce the response to medication with methylphenidate in ADHD. Therefore reasons for anxiety and adjustment issues have to be addressed and corrected early ${ }^{11}$. Patients who were treated with methylphenidate misused addictive substances less when they got older than the patients who were not prescribed it according to the metaanalytic review of the literature in the $\mathrm{West}^{11}$. In refractory patients with ADHD, the problem lies with the failure to follow medication and behavioural management. Contributors to this effect are doubtful public opinion about the efficacy of tablets to control hyperactivity in youngsters, social stigma and side effects to stimulant medication ${ }^{11}$.

\section{Conclusions}

- In this sample of 200 children with diagnosed ADHD, 139 (69.5\%) had one or more neuropsychiatric comorbidities.

- Specific developmental disorder of scholastic skills (SDDSS) was the most prevalent comorbidity in this study.

\section{References}

1. American Psychiatric Association( 2000) DSM $1 \mathrm{~V}:$ Diagnostic and Statistical Manual of Mental disorders iv text version 
2. Gelder MG, Andreasen NC, Ibor Jr. JJL Attention deficit hyperkinetic disorders in childhood and adolescence; New Oxford Textbook of Psychiatry: 1643-54; 2009

3. Thapar A, O'Donovan M, Owen MJ. The genetics of ADHD. Human Molecular Genetics 2005; 14, R 275-R282.

4. Klein RG, Mannuzza S. Long term outcome of hyperactive children;a review. Journal of American Academy of Child and Adolescent Psychiatry 1991; 29: 546-57. http://dx.doi.org/10.1097/00004583-19910500000005

5. Kariyawasam S H, Koralagama A, Jayawardane P, Karunathilake B, Perera V, Perera H. A descriptive study of attention deficit hyperactivity disorder (ADHD) at Lady Ridgeway Hospital for Children, Colombo Sri Lanka Journal of Child Health, 2002; 31: 109-14

6. Kessler RC, Adler L, Barkley R et al. Prevalence of adult ADHD in the US. Results from the National Comorbidity Survey Replication (NCSR) American Journal of Psychiatry 2006; 163: 716-23 http://dx.doi.org/10.1176/appi.ajp.163.4.716 PMid:16585449
7. Farone SV, Biderman J, Mick E. The age dependent decline of ADHD; a meta-analysis of follow-up studies. Psychological Medicine 2006; 36:159-65

http://dx.doi.org/10.1017/S003329170500471X PMid:16420712

8. Heptinstall E, Taylor E. Sex differences and their significance in ADHD of childhood (editor S .Sandberg). Cambridge University Press, Cambridge. 2002

9. Taylor E, Warner-Rogers J. Practitioner review; Early adversity and developmental disorders Journal of Child Psychology and Psychiatry 2005; 46,451-67

http://dx.doi.org/10.1111/j.14697610.2004.0040

2.x

PMid: 15845126

10. Ben Amor LL, Grizeznko N, Schwartz G, Lagiex $\mathrm{P}$, Baron C, Ter-Stepanian M, et al. Perinatal complications in children with attention deficit hyperactivity disorder and their unaffected siblings. Journal of Psychiatry and Neuroscience 2005; 30: 120-6.

PMid:15798787

11. Wilens TE, Faraone SV, Bidermann J. Does stimulant therapy of ADHD beget later substance abuse? Paediatrics 2003; 111: 179-85

http://dx.doi.org/10.1542/peds.111.1.179 